\title{
TWO-MODE OPTICAL TOMOGRAMS: A POSSIBLE EXPERIMENTAL CHECK OF THE ROBERTSON UNCERTAINTY RELATIONS
}

\author{
V.I. Man'ko ${ }^{a}$, G. Marmo ${ }^{b}$, A. Simoni ${ }^{b}$ and F. Ventriglia ${ }^{b}$ \\ ${ }^{a}$ P.N.Lebedev Physical Institute, Leninskii Prospect 53, Moscow 119991, Russia \\ (e-mail: manko@na.infn.it) \\ ${ }^{b}$ Dipartimento di Scienze Fisiche dell' Università "Federico II" e Sezione INFN di Napoli, \\ Complesso Universitario di Monte S. Angelo, via Cintia, 80126 Naples, Italy \\ (e-mail: marmo@na.infn.it, simoni@na.infn.it, ventriglia@na.infn.it)
}

September 22, 2018

\begin{abstract}
The experimental check of two-mode Robertson uncertainty relations and inequalities for highest quadrature moments is suggested by using homodyne photon detection. The relation between optical tomograms and symplectic tomograms is used to connect the tomographic dispersion matrix and the quadrature components dispersion matrix of the two-mode field states.
\end{abstract}

Keywords Optical tomogram, Robertson uncertainty relations.

PACS: 03.65-w, 03.65.Fd, 02.30.Uu

\section{Introduction}

Recently [1, 2], the possibility of experimentally checking of the SchrödingerRobertson uncertainty relations [3, 4] for conjugate position and momentum by using homodyne photon detection was suggested. 
The homodyne photon detection yields the optical tomogram [5, 6] of the photon quantum state and was implemented in the available experiments [7. 8, 9, 10, 11] to reconstruct the Wigner function of the state, which is interpreted as measuring the quantum state.

In probability representation of quantum mechanics [12, 13, 14, 15, 16] the state is identified with the tomographic probability which is either an optical [5, 6] or symplectic tomogram [12, 17] or other kinds of tomograms (see the review [18]).

In view of this, measuring the tomographic probability means to measure the quantum state, since the tomogram contains all the information on the quantum state including the information on the variances and covariances of the conjugate variables (position and momentum or field quadratures).

In this context, reconstructing the Wigner function for calculating other state properties like the quadrature dispersion matrix is useless, since all the characteristics can be obtained directly in terms of the measured tomographic probability distributions.

The aim of this work is to suggest possible experiments to obtain optical tomograms like for instance [11], where states of two-mode field were studied by measuring one-mode field states tomogram, and to check uncertainty relations for two-mode light.

The two-mode (multi-mode) uncertainty relations (see [4, 19, 20, 21]) seem to have been never checked directly in experiments.

Since the uncertainty relations are basic for quantum mechanics it is reasonable to have both experimental confirmation of their validity and knowledge of the experimental accuracy with which the uncertainty relations are checked.

Up to now all the tomographic approaches to measure quantum states were applied to one-mode light. Only recent experiments [11] were produced by homodyne detecting two-mode light. The experiments can be used to detect presence or absence of entanglement phenomena in the field under study.

In the two-mode case, the photon states which have been discussed the most have been assumed Gaussian. For Gaussian states the photon distribution function was obtained in explicit form [22] both for the one- and multi-mode cases in terms of Hermite polynomials depending on many variables.

One of our aims is to discuss the photon distribution in a two-mode field by means of the explicit expression given by the multi-variable Hermite poly- 
nomials in 22] for Gaussian light. This makes possible an additional control of both the Gaussianity property of the photon states and the accuracy of the measurements. We devote particular attention to the uncertainty relations in the tomographic probability representation for Gaussian states.

We also derive the tomographic form of the uncertainty relations for arbitrary quantum states to get formulae where the Robertson uncertainty relations are expressed in terms of two-mode symplectic tomograms which generalize the results of [1] obtained for one-mode states.

The paper is organized as follows. In section 2 we review the Robertson uncertainty relations. In section 3, we briefly review the tomographic probability representation of two-mode quantum states. In section 4, the marginal tomograms of two-mode probability distributions are discussed and the uncertainty relations for photon quadratures are given in the tomographic form appropriate for experimental study. Inequalities for the quadrature highest moments are given in section 5. Then state reconstruction is discussed in section 6 . The relations between quadratures and photon statistics are considered in section 7 . Conclusions and perspectives are drawn in section 8.

\section{Two-mode uncertainty relations}

The Robertson [23] uncertainty relations for two-mode systems read as a positivity condition for the matrix $\Sigma$ of the form:

$$
\Sigma=\left(\begin{array}{cccc}
\sigma_{P_{1} P_{1}} & \sigma_{P_{1} P_{2}} & \sigma_{P_{1} Q_{1}} & \sigma_{P_{1} Q_{2}} \\
\sigma_{P_{2} P_{1}} & \sigma_{P_{2} P_{2}} & \sigma_{P_{2} Q_{1}} & \sigma_{P_{2} Q_{2}} \\
\sigma_{Q_{1} P_{1}} & \sigma_{Q_{1} P_{2}} & \sigma_{Q_{1} Q_{1}} & \sigma_{Q_{1} Q_{2}} \\
\sigma_{Q_{2} P_{1}} & \sigma_{Q_{2} P_{2}} & \sigma_{Q_{2} Q_{1}} & \sigma_{Q_{2} Q_{2}}
\end{array}\right)+\frac{\mathrm{i}}{2}\left(\begin{array}{cccc}
0 & 0 & -1 & 0 \\
0 & 0 & 0 & -1 \\
1 & 0 & 0 & 0 \\
0 & 1 & 0 & 0
\end{array}\right) .
$$

The positivity condition for the above matrix

$$
\Sigma \geq 0
$$

means that all the principal minors of $\Sigma$ are non-negative. The dispersion matrix contribution into the matrix $\Sigma$ is positive (non-negative, in fact) both in classical and in quantum domain. The second contribution is due to noncommutativity of the conjugate variables in the quantum domain

$$
Q_{j} P_{k}-P_{k} Q_{j}=i \delta_{j k}
$$


Thus, one has the obvious inequalities

$$
\sigma_{P_{k} P_{k}} \geq 0 ; \sigma_{Q_{k} Q_{k}} \geq 0 ; k=1,2,
$$

accompanied by the Schrödinger-Robertson [4, 3] inequalities for each mode:

$$
\sigma_{P_{k} P_{k}} \sigma_{Q_{k} Q_{k}}-\sigma_{P_{k} Q_{k}}^{2}-\frac{1}{4} \geq 0 ; k=1,2
$$

Besides, there are inequalities which are cubic in variances and covariances, as:

$$
\operatorname{det}\left(\begin{array}{ccc}
\sigma_{P_{1} P_{1}} & \sigma_{P_{1} P_{2}} & \sigma_{P_{1} Q_{1}}-\frac{\mathrm{i}}{2} \\
\sigma_{P_{2} P_{1}} & \sigma_{P_{2} P_{2}} & \sigma_{P_{2} Q_{1}} \\
\sigma_{Q_{1} P_{1}}+\frac{\mathrm{i}}{2} & \sigma_{Q_{1} P_{2}} & \sigma_{Q_{1} Q_{1}}
\end{array}\right) \geq 0 .
$$

Also, one has a quartic inequality which is equivalent to the non-negativity of the 4-th principal minor of $\Sigma$ :

$$
\operatorname{det}\left(\begin{array}{cccc}
\sigma_{P_{1} P_{1}} & \sigma_{P_{1} P_{2}} & \sigma_{P_{1} Q_{1}} & \sigma_{P_{1} Q_{2}} \\
\sigma_{P_{2} P_{1}} & \sigma_{P_{2} P_{2}} & \sigma_{P_{2} Q_{1}} & \sigma_{P_{2} Q_{2}} \\
\sigma_{Q_{1} P_{1}} & \sigma_{Q_{1} P_{2}} & \sigma_{Q_{1} Q_{1}} & \sigma_{Q_{1} Q_{2}} \\
\sigma_{Q_{2} P_{1}} & \sigma_{Q_{2} P_{2}} & \sigma_{Q_{2} Q_{1}} & \sigma_{Q_{2} Q_{2}}
\end{array}\right) \geq \frac{1}{16}
$$

These inequalities can be checked by measuring the matrix elements of the dispersion matrix. This can be done in the tomographic approach.

\section{Tomograms - symplectic and optical}

The two-mode symplectic tomogram was introduced in [17]. Let us consider two homodyne quadratures

$$
\hat{X}_{1}=\mu_{1} Q_{1}+\nu_{1} P_{1} ; \hat{X}_{2}=\mu_{2} Q_{2}+\nu_{2} P_{2} .
$$

where the $Q$ 's and the $P$ 's are the usual position and momentum operators. The symplectic tomogram of a two mode density state $\hat{\rho}(1,2)$ depends on six real variables and reads: [18]

$$
\mathcal{M}\left(X_{1}, \mu_{1}, \nu_{1} ; X_{2}, \mu_{2}, \nu_{2}\right)=\operatorname{Tr}\left[\hat{\rho}(1,2) \delta\left(X_{1} \hat{I}-\hat{X}_{1}\right) \delta\left(X_{2} \hat{I}-\hat{X}_{2}\right)\right]
$$


In terms of Wigner function $W$ the symplectic tomogram reads:

$$
\begin{aligned}
& \mathcal{M}\left(X_{1}, \mu_{1}, \nu_{1} ; X_{2}, \mu_{2}, \nu_{2}\right)= \\
& \int W\left(q_{1}, p_{1} ; q_{2}, p_{2}\right) \delta\left(X_{1}-\mu_{1} q_{1}-\nu_{1} p_{1}\right) \delta\left(X_{2}-\mu_{2} q_{2}-\nu_{2} p_{2}\right) \frac{d q_{1} d p_{1} d q_{2} d p_{2}}{4 \pi^{2}} .
\end{aligned}
$$

and it is nonnegative and normalized:

$$
\int \mathcal{M}\left(X_{1}, \mu_{1}, \nu_{1} ; X_{2}, \mu_{2}, \nu_{2}\right) d X_{1} d X_{2}=1
$$

For

$$
\mu_{1}=\cos \theta_{1}, \nu_{1}=\sin \theta_{1} ; \mu_{2}=\cos \theta_{2}, \nu_{2}=\sin \theta_{2}
$$

one gets the optical two-mode tomogram depending on four essential real variables:

$$
\begin{aligned}
& \mathcal{W}\left(X_{1}, \theta_{1} ; X_{2}, \theta_{2}\right)= \\
& \mathcal{M}\left(X_{1}, \mu_{1}=\cos \theta_{1}, \nu_{1}=\sin \theta_{1} ; X_{2}, \mu_{2}=\cos \theta_{2}, \nu_{2}=\sin \theta_{2}\right) .
\end{aligned}
$$

This tomogram is the joint probability distribution of $X_{1}$ and $X_{2}$. In view of this one has

$$
\begin{aligned}
& \mathcal{W}^{(1)}\left(X_{1}, \theta_{1}\right)=\int \mathcal{W}\left(X_{1}, \theta_{1} ; X_{2}, \theta_{2}\right) d X_{2} ; \\
& \mathcal{W}^{(2)}\left(X_{2}, \theta_{2}\right)=\int \mathcal{W}\left(X_{1}, \theta_{1} ; X_{2}, \theta_{2}\right) d X_{1}
\end{aligned}
$$

where $\mathcal{W}^{(1)}\left(X_{1}, \theta_{1}\right)$ and $\mathcal{W}^{(2)}\left(X_{2}, \theta_{2}\right)$ are the optical tomograms of the first and second mode, respectively. In fact, $\mathcal{W}\left(X_{1}, \theta_{1} ; X_{2}, \theta_{2}\right)$ is a function of four variables, and integration over one variable would be expected to yield a function of three variables. On the contrary, the above formulae show that integration of the two-mode tomogram over a random variable $X_{k}$ gives a function independent of the associated variable parameter $\theta_{k}$. However, this property seems obvious in view of the physical meaning of a tomogram as joint probability density of two random position variables, measured in new reference frames in phase space, rotated by angles $\theta_{1}, \theta_{2}$. Since the tomogram of the first mode state is a marginal of the joint probability distribution, the integration over the second mode position washes out any information about the reference frame where the integrated position was measured. Such 
property takes place also for other tomographic probability distributions like spin tomograms of multi-qudit states [18].

It is worthy to note that for the Wigner function of a two-mode field, which is only a quasi-distribution function, such a property does not hold, so that one has to integrate over both conjugate position and momentum to obtain a one-mode Wigner function:

$$
\begin{aligned}
W^{(1)}\left(q_{1}, p_{1}\right) & =\int W\left(q_{1}, p_{1} ; q_{2}, p_{2}\right) \frac{d q_{2} d p_{2}}{2 \pi} \\
W^{(2)}\left(q_{2}, p_{2}\right) & =\int W\left(q_{1}, p_{1} ; q_{2}, p_{2}\right) \frac{d q_{1} d p_{1}}{2 \pi} .
\end{aligned}
$$

The one-mode tomograms $\mathcal{W}^{(k)}$ 's are related to the corresponding Wigner functions of the states as

$$
\mathcal{W}^{(k)}\left(X_{k}, \theta_{k}\right)=\int W^{(k)}\left(q_{k}, p_{k}\right) \delta\left(X_{k}-\cos \theta_{k} q_{k}-\sin \theta_{k} p_{k}\right) \frac{d q_{k} d p_{k}}{2 \pi},
$$

with $k=1,2$.

From Eq.(8) one obtains:

$$
\hat{X}_{k}^{2}=\mu_{k}^{2} Q_{k}^{2}+\nu_{k}^{2} P_{k}^{2}+2 \mu_{k} \nu_{k}\left(\frac{Q_{k} P_{k}+P_{k} Q_{k}}{2}\right) ;(k=1,2),
$$

and

$$
\hat{X}_{k} \hat{X}_{j}=\mu_{k} \mu_{j} Q_{k} Q_{j}+\nu_{k} \nu_{j} P_{k} P_{j}+\mu_{k} \nu_{j} Q_{k} P_{j}+\nu_{k} \mu_{j} P_{k} Q_{j} ;(j \neq k=1,2) .
$$

Due to the physical meaning of the tomogram as probability distribution, for homodyne quadratures one has

$$
\operatorname{Tr}\left[\hat{\rho}(1,2) \hat{X}_{k}^{n}\right]=\int X_{k}^{n} \mathcal{W}^{(k)}\left(X_{k}, \theta_{k}\right) d X_{k} ;(n=0,1,2,3, \ldots),
$$

and

$$
\operatorname{Tr}\left[\hat{\rho}(1,2) \hat{X}_{k} \hat{X}_{j}\right]=\int X_{k} X_{j} \mathcal{W}\left(X_{1}, \theta_{1} ; X_{2}, \theta_{2}\right) d X_{1} d X_{2} ;(j, k=1,2) .
$$

Bearing in mind the relations (16), (17), by means of the optical tomograms $\mathcal{W}\left(X_{1}, \theta_{1} ; X_{2}, \theta_{2}\right), \mathcal{W}^{(1)}\left(X_{1}, \theta_{1}\right), \mathcal{W}^{(2)}\left(X_{2}, \theta_{2}\right)$ one can express the matrix elements of the dispersion matrix of $\Sigma$ (eq. (10) in terms of integrals on 
the right hand side of the above equations (18), (19). In fact one gets:

$$
\sigma_{Q_{k} Q_{k}}=\int X_{k}^{2} \mathcal{W}^{(k)}\left(X_{k}, \theta_{k}=0\right) d X_{k}-\left(\int X_{k} \mathcal{W}^{(k)}\left(X_{k}, \theta_{k}=0\right) d X_{k}\right)^{2}
$$

and

$$
\sigma_{P_{k} P_{k}}=\int X_{k}^{2} \mathcal{W}^{(k)}\left(X_{k}, \theta_{k}=\frac{\pi}{2}\right) d X_{k}-\left(\int X_{k} \mathcal{W}^{(k)}\left(X_{k}, \theta_{k}=\frac{\pi}{2}\right) d X_{k}\right)^{2}
$$

with $k=1,2$. Moreover:

$$
\sigma_{Q_{k} P_{k}}=\sigma_{X_{k} X_{k}}\left(\frac{\pi}{4}\right)-\frac{1}{2} \sigma_{X_{k} X_{k}}(0)-\frac{1}{2} \sigma_{X_{k} X_{k}}\left(\frac{\pi}{2}\right) .
$$

One can see that it is possible to measure in terms of tomograms all the highest moments of the quadratures

$$
\left\langle Q_{k}^{n} P_{k}^{m}\right\rangle=\operatorname{Tr}\left[\hat{\rho}(1,2) Q_{k}^{n} P_{k}^{m}\right] ;(k=1,2) .
$$

For example, in the case of cubic moments, one has

$$
\left\langle Q_{k}^{3}\right\rangle=\int X_{k}^{3} \mathcal{W}^{(k)}\left(X_{k}, \theta_{k}=0\right) d X_{k} ;\left\langle P_{k}^{3}\right\rangle=\int X_{k}^{3} \mathcal{W}^{(k)}\left(X_{k}, \theta_{k}=\frac{\pi}{2}\right) d X_{k}
$$

By using

$$
\begin{aligned}
\hat{X}_{k}^{3} & =\mu_{k}^{3} Q_{k}^{3}+\nu_{k}^{3} P_{k}^{3}+\mu_{k}^{2} \nu_{k}\left(Q_{k}^{2} P_{k}+Q_{k} P_{k} Q_{k}+P_{k} Q_{k}^{2}\right) \\
& +\mu_{k} \nu_{k}^{2}\left(P_{k}^{2} Q_{k}+P_{k} Q_{k} P_{k}+Q_{k} P_{k}^{2}\right)
\end{aligned}
$$

and the commutation relations of the quadratures, one obtains

$$
Q_{k} P_{k} Q_{k}=P_{k} Q_{k}^{2}+i Q_{k} ; Q_{k}^{2} P_{k}=P_{k} Q_{k}^{2}+2 i Q_{k}
$$

and similarly

$$
P_{k} Q_{k} P_{k}=P_{k}^{2} Q_{k}+i P_{k} ; Q_{k} P_{k}^{2}=P_{k}^{2} Q_{k}+2 i P_{k}
$$

so that

$$
\hat{X}_{k}^{3}=\mu_{k}^{3} Q_{k}^{3}+\nu_{k}^{3} P_{k}^{3}+3 \mu_{k}^{2} \nu_{k}\left(P_{k} Q_{k}^{2}+i Q_{k}\right)+3 \mu_{k} \nu_{k}^{2}\left(P_{k}^{2} Q_{k}+i P_{k}\right) .
$$




\section{Marginals of two-mode tomograms}

Let us consider light modes which can be obtained by means of optical devices from two initial ones described by operators $a$ and $b$ satisfying the commutation relations

$$
\left[a, a^{\dagger}\right]=\left[b, b^{\dagger}\right]=1 ;[a, b]=\left[a, b^{\dagger}\right]=\left[a^{\dagger}, b\right]=0 .
$$

Then one can make symplectic transformations and get the modes described by the operators

$$
\begin{aligned}
& c=\frac{1}{\sqrt{2}}(a+b) ; d=\frac{1}{\sqrt{2}}(a-b) ; \\
& e=\frac{1}{\sqrt{2}}(a+\mathrm{i} b) ; f=\frac{1}{\sqrt{2}}(a-\mathrm{i} b) .
\end{aligned}
$$

One can readily check that

$$
\left[c, c^{\dagger}\right]=\left[d, d^{\dagger}\right]=\left[e, e^{\dagger}\right]=\left[f, f^{\dagger}\right]=1 .
$$

The initial modes can be expressed in terms of quadratures:

$$
a=\frac{1}{\sqrt{2}}\left(Q_{1}+\mathrm{i} P_{1}\right) ; b=\frac{1}{\sqrt{2}}\left(Q_{2}+\mathrm{i} P_{2}\right)
$$

Besides, one has homodyne quadrature operators for each of these modes

$$
\hat{X}_{a}\left(\mu_{1}, \nu_{1}\right)=\left(\mu_{1} Q_{1}+\nu_{1} P_{1}\right) ; \hat{X}_{b}\left(\mu_{2}, \nu_{2}\right)=\mu_{2} Q_{2}+\nu_{2} P_{2} .
$$

where one can take local oscillator phases to set

$$
\mu_{1}=\cos \theta_{1}, \nu_{1}=\sin \theta_{1} ; \mu_{2}=\cos \theta_{2}, \nu_{2}=\sin \theta_{2} .
$$

Then one can consider the homodyne quadrature operators for all four modes given by (30) in terms of initial quadratures operators

$$
\begin{aligned}
\hat{X}_{c}\left(\mu_{3}, \nu_{3}\right) & =\frac{1}{2} \mu_{3}\left(Q_{1}+Q_{2}\right)+\frac{1}{2} \nu_{3}\left(P_{1}+P_{2}\right) \\
\hat{X}_{d}\left(\mu_{4}, \nu_{4}\right) & =\frac{1}{2} \mu_{4}\left(Q_{1}-Q_{2}\right)+\frac{1}{2} \nu_{4}\left(P_{1}-P_{2}\right) \\
\hat{X}_{e}\left(\mu_{5}, \nu_{5}\right) & =\frac{1}{2} \mu_{5}\left(Q_{1}-P_{2}\right)+\frac{1}{2} \nu_{5}\left(Q_{2}+P_{1}\right) \\
\hat{X}_{f}\left(\mu_{6}, \nu_{6}\right) & =\frac{1}{2} \mu_{6}\left(Q_{1}+P_{2}\right)+\frac{1}{2} \nu_{6}\left(P_{1}-Q_{2}\right)
\end{aligned}
$$


We now shift from labels $a, b, c, d, e, f$ to labels $1,2,3,4,5,6$, respectively, so that $\hat{X}_{a}\left(\mu_{1}, \nu_{1}\right) \rightarrow \hat{X}_{1}\left(\mu_{1}, \nu_{1}\right), \hat{X}_{b}\left(\mu_{2}, \nu_{2}\right) \rightarrow \hat{X}_{2}\left(\mu_{2}, \nu_{2}\right)$ and so on. In the above equations the parameters may be chosen as

$$
\mu_{k}=\cos \theta_{k}, \nu_{k}=\sin \theta_{k},(k=3,4,5,6) .
$$

The above equations (35) can be given a vector form:

$$
\hat{\boldsymbol{X}}=S \boldsymbol{Q}
$$

where the vector $\hat{\boldsymbol{X}}, \boldsymbol{Q}$ have operator components $\hat{X}_{3}, \hat{X}_{4}, \hat{X}_{5}, \hat{X}_{6}$ and $P_{1}, P_{2}, Q_{1}, Q_{2}$ respectively, while the matrix $S$ reads

$$
S=\frac{1}{2}\left(\begin{array}{cccc}
\nu_{3} & \nu_{3} & \mu_{3} & \mu_{3} \\
\nu_{4} & -\nu_{4} & \mu_{4} & -\mu_{4} \\
\nu_{5} & -\mu_{5} & \mu_{5} & \nu_{5} \\
\nu_{6} & \mu_{6} & \nu_{6} & -\mu_{6}
\end{array}\right)
$$

and is invertible, as det $S \neq 0$ in the generic case. So one can solve with respect to $\boldsymbol{Q}$ and obtain

$$
Q=S^{-1} \hat{\boldsymbol{X}}
$$

or, taking mean values:

$$
\langle\boldsymbol{Q}\rangle=S^{-1}\langle\hat{\boldsymbol{X}}\rangle
$$

where operators are averaged in the density state $\rho:\langle\hat{A}\rangle:=\operatorname{Tr}(\rho \hat{A})$.

One-mode homodyne detectors can measure the six optical tomograms

$$
\mathcal{W}_{k}\left(X_{k}, \theta_{k}\right)=\operatorname{Tr}\left(\rho \hat{X}_{k}\right),(k=1,2, \ldots, 6)
$$

For each $k$-mode one has the Schrödinger-Robertson inequality expressed in 
terms of measured tomograms [1] associated with the two-mode light state

$$
\begin{aligned}
F\left(\theta_{k}\right)= & \left(\int X_{k}^{2} \mathcal{W}_{k}\left(X_{k}, \theta_{k}\right) d X_{k}-\left[\int X_{k} \mathcal{W}_{k}\left(X_{k}, \theta_{k}\right) d X_{k}\right]^{2}\right) \\
& \left(\int X_{k}^{2} \mathcal{W}_{k}\left(X_{k}, \theta_{k}+\frac{\pi}{2}\right) d X_{k}-\left[\int X_{k} \mathcal{W}_{k}\left(X_{k}, \theta_{k}+\frac{\pi}{2}\right) d X_{k}\right]^{2}\right. \\
& -\left\{\int X_{k}^{2} \mathcal{W}_{k}\left(X_{k}, \theta_{k}+\frac{\pi}{4}\right) d X_{k}-\left[\int X_{k} \mathcal{W}_{k}\left(X_{k}, \theta_{k}+\frac{\pi}{4}\right) d X_{k}\right]^{2}\right. \\
& -\frac{1}{2}\left[\int X_{k}^{2} \mathcal{W}_{k}\left(X_{k}, \theta_{k}\right) d X_{k}-\left[\int X_{k} \mathcal{W}_{k}\left(X_{k}, \theta_{k}\right) d X_{k}\right]^{2}\right. \\
& \left.\left.+\int X_{k}^{2} \mathcal{W}_{k}\left(X_{k}, \theta_{k}+\frac{\pi}{2}\right) d X_{k}-\left[\int X_{k} \mathcal{W}_{k}\left(X_{k}, \theta_{k}+\frac{\pi}{2}\right) d X_{k}\right]^{2}\right]\right\}^{2}-\frac{1}{4} \geq 0 .
\end{aligned}
$$

From the homodyne quadrature operators (33) one gets:

$$
Q_{1}=\hat{X}_{1}(1,0), P_{1}=\hat{X}_{1}(0,1) ; Q_{2}=\hat{X}_{2}(1,0), P_{2}=\hat{X}_{2}(0,1) ;
$$

Moreover:

$$
\begin{aligned}
Q_{1}^{2} & =\hat{X}_{1}^{2}(1,0), P_{1}^{2}=\hat{X}_{1}^{2}(0,1) \\
\frac{1}{2}\left\{Q_{1}, P_{1}\right\} & =\hat{X}_{1}^{2}\left(\frac{\sqrt{2}}{2}, \frac{\sqrt{2}}{2}\right)-\frac{1}{2}\left[\hat{X}_{1}^{2}(1,0)+\hat{X}_{1}^{2}(0,1)\right]
\end{aligned}
$$

and analogously, turning label 1 into label 2 :

$$
\begin{aligned}
Q_{2}^{2} & =\hat{X}_{2}^{2}(1,0), P_{2}^{2}=\hat{X}_{2}^{2}(0,1) \\
\frac{1}{2}\left\{Q_{2}, P_{2}\right\} & =\hat{X}_{2}^{2}\left(\frac{\sqrt{2}}{2}, \frac{\sqrt{2}}{2}\right)-\frac{1}{2}\left[\hat{X}_{2}^{2}(1,0)+\hat{X}_{2}^{2}(0,1)\right] .
\end{aligned}
$$

Also:

$$
\begin{aligned}
Q_{1} Q_{2} & =2 \hat{X}_{3}^{2}(1,0)-\frac{1}{2}\left[\hat{X}_{1}^{2}(1,0)+\hat{X}_{2}^{2}(1,0)\right] \\
P_{1} P_{2} & =2 \hat{X}_{3}^{2}(0,1)-\frac{1}{2}\left[\hat{X}_{1}^{2}(0,1)+\hat{X}_{2}^{2}(0,1)\right]
\end{aligned}
$$


and finally

$$
\begin{aligned}
Q_{1} P_{2} & =-2 \hat{X}_{5}^{2}(1,0)+\frac{1}{2}\left[\hat{X}_{1}^{2}(1,0)+\hat{X}_{2}^{2}(0,1)\right] ; \\
Q_{2} P_{1} & =2 \hat{X}_{5}^{2}(0,1)-\frac{1}{2}\left[\hat{X}_{1}^{2}(0,1)+\hat{X}_{2}^{2}(1,0)\right] .
\end{aligned}
$$

The obtained equalities allow to express the Robertson uncertainty relations in terms of homodyne tomograms which are experimentally measured. There are also relations which are compatible with the properties of the different homodyne quadratures (33), (35), for example

$$
\hat{X}_{3}(1,0)=\frac{1}{2}\left[\hat{X}_{1}(1,0)+\hat{X}_{2}(1,0)\right]
$$

and many other including quadrature equalities.

The expressions for variances and covariances of the two-mode field quadrature components are, with $k=1,2$ :

$$
\begin{aligned}
\sigma_{Q_{k} Q_{k}} & =\left(\int X_{k}^{2} \mathcal{W}_{k}\left(X_{k}, \theta_{k}=0\right) d X_{k}-\left[\int X_{k} \mathcal{W}_{k}\left(X_{k}, \theta_{k}=0\right) d X_{k}\right]^{2}\right) \\
\sigma_{P_{k} P_{k}} & =\left(\int X_{k}^{2} \mathcal{W}_{k}\left(X_{k}, \theta_{k}=\frac{\pi}{2}\right) d X_{k}-\left[\int X_{k} \mathcal{W}_{k}\left(X_{k}, \theta_{k}=\frac{\pi}{2}\right) d X_{k}\right]^{2}\right) \\
\sigma_{Q_{k} P_{k}} & =\left(\int X_{k}^{2} \mathcal{W}_{k}\left(X_{k}, \theta_{k}=\frac{\pi}{4}\right) d X_{k}-\left[\int X_{k} \mathcal{W}_{k}\left(X_{k}, \theta_{k}=\frac{\pi}{4}\right) d X_{k}\right]^{2}\right) \\
& -\frac{1}{2}\left(\sigma_{Q_{k} Q_{k}}+\sigma_{P_{k} P_{k}}\right) .
\end{aligned}
$$

The same expressions appear in formula (41) defining $F\left(\theta_{k}\right)$. Besides, in view of (45) one has

$$
\begin{aligned}
\sigma_{Q_{1} Q_{2}} & =2\left(\int X_{3}^{2} \mathcal{W}_{3}\left(X_{3}, \theta_{3}=0\right) d X_{3}-\left[\int X_{3} \mathcal{W}_{3}\left(X_{3}, \theta_{3}=0\right) d X_{3}\right]^{2}\right) \\
& -\frac{1}{2}\left(\sigma_{Q_{1} Q_{1}}+\sigma_{Q_{2} Q_{2}}\right) \\
\sigma_{P_{1} P_{2}} & =2\left(\int X_{3}^{2} \mathcal{W}_{3}\left(X_{3}, \theta_{3}=\frac{\pi}{2}\right) d X_{3}-\left[\int X_{3} \mathcal{W}_{3}\left(X_{3}, \theta_{3}=\frac{\pi}{2}\right) d X_{3}\right]^{2}\right) \\
& -\frac{1}{2}\left(\sigma_{P_{1} P_{1}}+\sigma_{P_{2} P_{2}}\right),
\end{aligned}
$$


and finally, from (46) one gets

$$
\begin{aligned}
\sigma_{Q_{1} P_{2}} & =-2\left(\int X_{5}^{2} \mathcal{W}_{5}\left(X_{5}, \theta_{5}=0\right) d X_{5}-\left[\int X_{5} \mathcal{W}_{5}\left(X_{5}, \theta_{5}=0\right) d X_{5}\right]^{2}\right) \\
& +\frac{1}{2}\left(\sigma_{Q_{1} Q_{1}}+\sigma_{P_{2} P_{2}}\right) ; \\
\sigma_{Q_{2} P_{1}} & =2\left(\int X_{5}^{2} \mathcal{W}_{5}\left(X_{5}, \theta_{5}=\frac{\pi}{2}\right) d X_{5}-\left[\int X_{5} \mathcal{W}_{5}\left(X_{5}, \theta_{5}=\frac{\pi}{2}\right) d X_{5}\right]^{2}\right) \\
& -\frac{1}{2}\left(\sigma_{Q_{2} Q_{2}}+\sigma_{P_{1} P_{1}}\right) .
\end{aligned}
$$

Inserting the obtained variances and covariances into the matrix $\Sigma$ defined in Eq.(1) one can calculate all its principal minors in terms of the measured tomograms $\mathcal{W}_{k}\left(X_{k}, \theta_{k}\right)$ and check by direct experimental data the Robertson uncertainty relations by means of the positivity of such minors. One can tell that the experimental data for all six modes are redundant to get the dispersion matrix for the quadratures. One could use other mode combinations. For example, we did not use the mode data associated with the tomogram $\mathcal{W}_{6}\left(X_{6}, \theta_{6}\right)$. Another set of tomograms could be used to get the dispersion matrix. This variety is useful to make extra control of accuracy of the measurements because the dispersion must be the same irrespectively of the particular set of tomograms used. Thus one has the dispersion matrix with "commutator" contributions in different permutations of basis, e.g.

$$
\Sigma=\left(\begin{array}{crcc}
\sigma_{P_{1} P_{1}} & \sigma_{P_{1} P_{2}} & \sigma_{P_{1} Q_{1}}-\frac{\mathrm{i}}{2} & \sigma_{P_{1} Q_{2}} \\
\sigma_{P_{2} P_{1}} & \sigma_{P_{2} P_{2}} & \sigma_{P_{2} Q_{1}} & \sigma_{P_{2} Q_{2}}-\frac{\mathrm{i}}{2} \\
\sigma_{Q_{1} P_{1}}+\frac{\mathrm{i}}{2} & \sigma_{Q_{1} P_{2}} & \sigma_{Q_{1} Q_{1}} & \sigma_{Q_{1} Q_{2}} \\
\sigma_{Q_{2} P_{1}} & \sigma_{Q_{2} P_{2}}+\frac{\mathrm{i}}{2} & \sigma_{Q_{2} Q_{1}} & \sigma_{Q_{2} Q_{2}}
\end{array}\right)
$$

and

$$
\Sigma^{\prime}=\left(\begin{array}{cccc}
\sigma_{Q_{1} Q_{1}} & \sigma_{Q_{1} Q_{2}} & \sigma_{Q_{1} P_{1}}+\frac{\mathrm{i}}{2} & \sigma_{Q_{1} P_{2}} \\
\sigma_{Q_{2} Q_{1}} & \sigma_{Q_{2} Q_{2}} & \sigma_{Q_{2} P_{1}} & \sigma_{Q_{2} P_{2}}+\frac{\mathrm{i}}{2} \\
\sigma_{P_{1} Q_{1}}-\frac{\mathrm{i}}{2} & \sigma_{P_{1} Q_{2}} & \sigma_{P_{1} P_{1}} & \sigma_{P_{1} P_{2}} \\
\sigma_{P_{2} Q_{1}} & \sigma_{P_{2} Q_{2}}-\frac{\mathrm{i}}{2} & \sigma_{P_{2} P_{1}} & \sigma_{P_{2} P_{2}}
\end{array}\right) .
$$

All the elements of the matrices are expressed in terms of measured tomograms. One has to check the non-negativity of the principal minors. Although theoretically the non-negativity of the principal minors found in one basis induces their nonnegativity in all of the other bases, the checking of the 
experimental data looks different since the order of the checking depends on the basis. For example, in the case of the matrix $\Sigma$ the second principal minor provides a checking of the Schrödinger-Robertson uncertainty relations, while using $\Sigma^{\prime}$ the second leading principal minor

$$
M_{2}=\sigma_{Q_{1} Q_{1}} \sigma_{Q_{2} Q_{2}}-\sigma_{Q_{1} Q_{2}}^{2} \geq 0
$$

one checks a classical property which does not contain Planck's constant influence. Thus, to check the uncertainty relations and to control the accuracy of the data, it seems to be reasonable to check the non-negativity of the principal minors in all the bases.

The non-negativity of the third leading principal minor yields the constraints obtained from the matrices $\Sigma$ and $\Sigma^{\prime}$ respectively:

$$
\operatorname{det}\left(\begin{array}{ccc}
\sigma_{P_{1} P_{1}} & \sigma_{P_{1} P_{2}} & \sigma_{P_{1} Q_{1}}-\frac{\mathrm{i}}{2} \\
\sigma_{P_{2} P_{1}} & \sigma_{P_{2} P_{2}} & \sigma_{P_{2} Q_{1}} \\
\sigma_{Q_{1} P_{1}}+\frac{\mathrm{i}}{2} & \sigma_{Q_{1} P_{2}} & \sigma_{Q_{1} Q_{1}}
\end{array}\right) \geq 0
$$

and

$$
\operatorname{det}\left(\begin{array}{ccc}
\sigma_{Q_{1} Q_{1}} & \sigma_{Q_{1} Q_{2}} & \sigma_{Q_{1} P_{1}}+\frac{\mathrm{i}}{2} \\
\sigma_{Q_{2} Q_{1}} & \sigma_{Q_{2} Q_{2}} & \sigma_{Q_{2} P_{1}} \\
\sigma_{P_{1} Q_{1}}-\frac{\mathrm{i}}{2} & \sigma_{P_{1} Q_{2}} & \sigma_{P_{1} P_{1}}
\end{array}\right) \geq 0 .
$$

The last minor is just the determinant of the matrix $\Sigma$, or $\Sigma^{\prime}$.

\section{$5 \quad$ Measuring highest moments of quadratures by homodyne detector}

Let us discuss first how to measure highest moments for one-mode light, say the $a$-mode given by Eq.(33), $\hat{X}_{1}\left(\mu_{1}, \nu_{1}\right)=\mu_{1} Q_{1}+\nu_{1} P_{1}$. Mean values, variances and covariances are given in terms of the tomogram $\mathcal{W}_{1}\left(X_{1}, \theta_{1}\right)$. Let us construct cubic moments. Then, dropping label 1, one has to find the moments of the operators $P^{3}, P^{2} Q, P Q^{2}, Q^{3}$ because the remaining may be expressed by commutators as

$$
\begin{aligned}
& P Q P=P P Q+P[Q, P]=P^{2} Q+\mathrm{i} P \Rightarrow\langle P Q P\rangle=\left\langle P^{2} Q\right\rangle+\mathrm{i}\langle P\rangle,(52) \\
& Q P P=P Q P+[Q, P] P=P^{2} Q+2 \mathrm{i} P \Rightarrow\left\langle Q P^{2}\right\rangle=\left\langle P^{2} Q\right\rangle+2 \mathrm{i}\langle P\rangle,
\end{aligned}
$$


and analogously

$$
\begin{aligned}
\langle Q P Q\rangle & =\left\langle P Q^{2}\right\rangle+\mathrm{i}\langle Q\rangle, \\
\left\langle Q^{2} P\right\rangle & =\left\langle P Q^{2}\right\rangle+2 \mathrm{i}\langle Q\rangle .
\end{aligned}
$$

The cubic power $\hat{X}^{3}(\mu, \nu)$ reads

$$
\begin{aligned}
\hat{X}^{3}(\mu, \nu) & =\mu^{3} Q^{3}+\nu^{3} P^{3}+\mu^{2} \nu\left(Q^{2} P+Q P Q+P Q^{2}\right) \\
& +\mu \nu^{2}\left(P^{2} Q+P Q P+Q P^{2}\right)
\end{aligned}
$$

so that

$$
\begin{aligned}
\left\langle\hat{X}^{3}\right\rangle(\mu, \nu) & =\mu^{3}\left\langle Q^{3}\right\rangle+\nu^{3}\langle P\rangle^{3}+3 \mu^{2} \nu\left(\left\langle P Q^{2}\right\rangle+i\langle Q\rangle\right) \\
& +3 \mu \nu^{2}\left(\left\langle P^{2} Q\right\rangle+i\langle P\rangle\right) .
\end{aligned}
$$

The means of the quadratures read

$$
\langle\hat{X}\rangle(1,0)=\langle Q\rangle,\langle\hat{X}\rangle(0,1)=\langle P\rangle .
$$

Besides, one has

$$
\left\langle\hat{X}^{3}\right\rangle(1,0)=\left\langle Q^{3}\right\rangle,\left\langle\hat{X}^{3}\right\rangle(0,1)=\left\langle P^{3}\right\rangle
$$

and

$$
\begin{aligned}
\left\langle\hat{X}^{3}\right\rangle(\mu, \nu) & =\mu^{3}\left\langle\hat{X}^{3}\right\rangle(1,0)+\nu^{3}\left\langle\hat{X}^{3}\right\rangle(0,1)+3 \mu^{2} \nu\left(\left\langle P Q^{2}\right\rangle+i\langle\hat{X}\rangle\right. \\
& +3 \mu \nu^{2}\left(\left\langle P^{2} Q\right\rangle+i\langle\hat{X}\rangle(0,1)\right) .
\end{aligned}
$$

Introducing the function

$$
\begin{aligned}
A(\mu, \nu): & =\left\langle\hat{X}^{3}\right\rangle(\mu, \nu)-\mu^{3}\left\langle\hat{X}^{3}\right\rangle(1,0)-\nu^{3}\left\langle\hat{X}^{3}\right\rangle(0,1) \\
& -3 \mu^{2} \nu i\langle\hat{X}\rangle(1,0)-3 \mu \nu^{2} i\langle\hat{X}\rangle(0,1)
\end{aligned}
$$

we obtain two linear equations for the remaining two moments:

$$
\begin{aligned}
& A\left(\mu_{\alpha}, \nu_{\alpha}\right)=3 \mu_{\alpha}^{2} \nu_{\alpha}\left\langle P Q^{2}\right\rangle+3 \mu_{\alpha} \nu_{\alpha}^{2}\left\langle P^{2} Q\right\rangle ; \\
& A\left(\mu_{\beta}, \nu_{\beta}\right)=3 \mu_{\beta}^{2} \nu_{\beta}\left\langle P Q^{2}\right\rangle+3 \mu_{\beta} \nu_{\beta}^{2}\left\langle P^{2} Q\right\rangle ;
\end{aligned}
$$


which can be readily solved in terms of the homodyne quadratures given by the tomogram $\mathcal{W}(X, \theta)$ only.

The previous construction of the solutions

$$
\left\langle P Q^{2}\right\rangle=\frac{1}{\Delta} \operatorname{det}\left(\begin{array}{ll}
A\left(\mu_{\alpha}, \nu_{\alpha}\right) & 3 \mu_{\alpha} \nu_{\alpha}^{2} \\
A\left(\mu_{\beta}, \nu_{\beta}\right) & 3 \mu_{\beta} \nu_{\beta}^{2}
\end{array}\right) ;\left\langle P^{2} Q\right\rangle=\frac{1}{\Delta} \operatorname{det}\left(\begin{array}{cc}
3 \mu_{\alpha}^{2} \nu_{\alpha} & A\left(\mu_{\alpha}, \nu_{\alpha}\right) \\
3 \mu_{\beta}^{2} \nu_{\beta} & A\left(\mu_{\beta}, \nu_{\beta}\right)
\end{array}\right),
$$

with

$$
\Delta=\operatorname{det}\left(\begin{array}{cc}
3 \mu_{\alpha}^{2} \nu_{\alpha} & 3 \mu_{\alpha} \nu_{\alpha}^{2} \\
3 \mu_{\beta}^{2} \nu_{\beta} & 3 \mu_{\beta} \nu_{\beta}^{2}
\end{array}\right),
$$

shows that the same procedure can be applied to get all the highest moments $\left\langle P^{n} Q^{m}\right\rangle$ and $\left\langle P^{m} Q^{n}\right\rangle \quad(n, m=0,1, \ldots)$ in terms of the tomogram $\mathcal{W}(X, \theta)$ only. It provides the tool to check all the known high moments quantum uncertainty relations [19] in fact both in one mode and multi-mode case. As an example we derive simple uncertainty relations for cubic moments.

Let us consider the linear forms:

$$
\hat{f}=y_{1} Q+y_{2} P^{2} ; \hat{f}^{\dagger}=y_{1}^{*} Q+y_{2}^{*} P^{2} .
$$

The obvious inequality for the mean value

$$
\left\langle\hat{f} \hat{f}^{\dagger}\right\rangle \geq 0
$$

gives a condition of nonnegativity for the quadratic form

$$
y_{1} y_{1}^{*}\left\langle Q^{2}\right\rangle+y_{1} y_{2}^{*}\left\langle Q P^{2}\right\rangle+y_{2} y_{1}^{*}\left\langle P^{2} Q\right\rangle+y_{2} y_{2}^{*}\left\langle P^{4}\right\rangle \geq 0 .
$$

Thus the matrix of the quadratic form

$$
M=\left(\begin{array}{cc}
\left\langle Q^{2}\right\rangle & \left\langle Q P^{2}\right\rangle \\
\left\langle P^{2} Q\right\rangle & \left\langle P^{4}\right\rangle
\end{array}\right)
$$

must be nonnegative, and this implies

$$
\left\langle Q^{2}\right\rangle\left\langle P^{4}\right\rangle-\left\langle Q P^{2}\right\rangle\left\langle P^{2} Q\right\rangle \geq 0
$$

This inequality can be written in terms of tomograms as

$$
\int X^{2} \mathcal{W}(X, \theta=0) d X \int X^{4} \mathcal{W}\left(X, \theta=\frac{\pi}{2}\right) d X-\left[\left\langle Q P^{2}\right\rangle\left\langle P^{2} Q\right\rangle\right]_{\theta_{\alpha}, \theta_{\beta}} \geq 0(68)
$$


where local oscillator phases, for instance $\theta_{\alpha}=\pi / 3, \theta_{\beta}=2 \pi / 3$, are taken ìn Eq. (61), so that the parameters $\left(\mu_{\alpha}, \nu_{\alpha}\right)$ and $\left(\mu_{\beta}, \nu_{\beta}\right)$ are $(\sqrt{3} / 2,1 / 2)$ and $(1 / 2, \sqrt{3} / 2)$ respectively. Of course, one could use other suitable local oscillator phases, such that $\Delta \neq 0$ in Eq. (61). The above cubic-in-quadrature uncertainty relation must be satisfied by any of the six modes used in experiments [11].

In view of the generalization proposed in [1] for Schrödinger-Robertson uncertainty relations, an analogous generalization can be proposed for the above highest order moments inequality, that can be written in covariant form, i.e. for all the local oscillator phases as:

$$
\begin{aligned}
& \int X^{2} \mathcal{W}(X, \theta) d X \int X^{4} \mathcal{W}\left(X, \theta+\frac{\pi}{2}\right) d X \\
& -\left[\left\langle Q P^{2}\right\rangle\left\langle P^{2} Q\right\rangle\right]_{\theta+\theta_{\alpha}, \theta+\theta_{\beta}} \geq 0,
\end{aligned}
$$

where, as before, Eq. (61) has to be used with the new values of local oscillator phases, say $\theta+\pi / 3, \theta+2 \pi / 3$.

\section{State reconstruction}

The one-mode measurement can be used to get complete information on the two-mode state. In fact, the complete information is contained in the symplectic tomogram $\mathcal{M}\left(X_{1}, \mu_{1}, \nu_{1} ; X_{2}, \mu_{2}, \nu_{2}\right)$ or in the optical tomogram $\mathcal{W}\left(X_{1}, \theta_{1} ; X_{2}, \theta_{2}\right)$. The same information on the state is contained in the Wigner function $W\left(q_{1}, p_{1} ; q_{2}, p_{2}\right)$.

We recall that one-mode states have tomogram

$$
\mathcal{W}^{(1)}\left(X_{1}, \theta_{1}\right)=\int \mathcal{W}\left(X_{1}, \theta_{1} ; X_{2}, \theta_{2}\right) d X_{2}
$$

and Wigner function

$$
W^{(1)}\left(q_{1}, p_{1}\right)=\int W\left(q_{1}, p_{1} ; q_{2}, p_{2}\right) \frac{d q_{2} d p_{2}}{2 \pi} .
$$

A natural question to ask: is it possible to find the two-mode state either in terms of tomogram or in terms of Wigner function, if only one-mode tomograms can be measured? The answer is positive, in fact. It was shown 
24 that a symplectic transformation $V$ acts on Wigner function or tomogram of the anti-transformed state $V \rho V^{\dagger}$. In experiment [11] the one-mode tomograms are measured for such symplectically transformed states. Thus one has the marginal probability distributions depending on a set of parameters sufficient to find out the two-mode state tomogram (Wigner function or density operator).

This property may be understood in general terms. The quadratures of multi-mode field close on the Lie algebra of the Weyl-Heisenberg group. All the highest quadrature moments are determined by the elements of the enveloping of this Weyl-Heisenberg algebra. Then, any new basis of the Lie algebra obtained by the initial one by a linear invertible transformation gives rise to the same enveloping algebra. So, having new transformed modes and measuring the corresponding one-mode tomograms, makes possible to find out all the highest moments of the multi-mode field. This means that one can reconstruct the multi-mode state tomogram by measuring only a set of suitably chosen one-mode tomograms. We now show this procedure on the example of a two-mode field.

The idea of reconstructing the two-mode density operator by measuring several one-mode density operators is the following one. The marginals give us the possibility to find all the quadrature moments of two-mode light in terms of one-mode tomograms only. Then the Wigner function, tomogram or density operator are expressed in terms of moments. For example the characteristic function of the tomogram, which is the Fourier transform of the tomogram, reads

$$
\begin{aligned}
\tilde{\mathcal{W}}\left(K_{1}, \theta_{1} ; K_{2}, \theta_{2}\right) & :=\int \mathcal{W}\left(X_{1}, \theta_{1} ; X_{2}, \theta_{2}\right) \exp \left[\mathrm{i}\left(K_{1} X_{1}+K_{2} X_{2}\right)\right] d X_{1} d X_{2} \\
& =\sum_{n, m=0}^{\infty} \frac{\left(\mathrm{i} K_{1}\right)^{n}\left(\mathrm{i} K_{2}\right)^{m}}{n ! m !}\left\langle X_{1}^{n} X_{2}^{m}\right\rangle\left(\theta_{1}, \theta_{2}\right)
\end{aligned}
$$

where the moments explicitly are:

$$
\left\langle X_{1}^{n} X_{2}^{m}\right\rangle\left(\theta_{1}, \theta_{2}\right)=\int X_{1}^{n} X_{2}^{m} \mathcal{W}\left(X_{1}, \theta_{1} ; X_{2}, \theta_{2}\right) d X_{1} d X_{2}
$$

The knowledge of all moments allows the reconstruction of the characteristic function. Then the tomogram is given by a Fourier anti-transform:.

$$
\mathcal{W}\left(X_{1}, \theta_{1} ; X_{2}, \theta_{2}\right)=\int \tilde{\mathcal{W}}\left(K_{1}, \theta_{1} ; K_{2}, \theta_{2}\right) \exp \left[-\mathrm{i}\left(K_{1} X_{1}+K_{2} X_{2}\right)\right] \frac{d K_{1} d K_{2}}{(2 \pi)^{2}} .
$$


Analogously, the Wigner function of the two-mode state is known as soon as the moments

$$
\left\langle q_{1}^{n} p_{1}^{m} q_{2}^{n^{\prime}} p_{2}^{m^{\prime}}\right\rangle=\int q_{1}^{n} p_{1}^{m} q_{2}^{n^{\prime}} p_{2}^{m^{\prime}} W\left(q_{1}, p_{1} ; q_{2}, p_{2}\right) \frac{d q_{1} d p_{1} d q_{2} d p_{2}}{(2 \pi)^{2}}
$$

are known. Again, these moments determine the characteristic function:

$$
\begin{aligned}
& \tilde{W}\left(\xi_{1}, \eta_{1} ; \xi_{2}, \eta_{2}\right) \\
:= & \int W\left(q_{1}, p_{1} ; q_{2}, p_{2}\right) \exp \left[\mathrm{i}\left(\xi_{1} q_{1}+\eta_{1} p_{1}+\xi_{2} q_{2}+\eta_{2} p_{2}\right)\right] \frac{d q_{1} d p_{1} d q_{2} d p_{2}}{(2 \pi)^{2}} \\
= & \sum_{n, m, n^{\prime}, m^{\prime}=0}^{\infty} \frac{\left(\mathrm{i} \xi_{1}\right)^{n}\left(\mathrm{i} \eta_{1}\right)^{m}\left(\mathrm{i} \xi_{2}\right)^{n^{\prime}}\left(\mathrm{i} \eta_{2}\right)^{m^{\prime}}}{n ! m ! n^{\prime} ! m^{\prime} !}\left\langle q_{1}^{n} p_{1}^{m} q_{2}^{n^{\prime}} p_{2}^{m^{\prime}}\right\rangle
\end{aligned}
$$

and the Wigner function is obtained by anti-Fourier transforming:

$$
\begin{aligned}
& W\left(q_{1}, p_{1} ; q_{2}, p_{2}\right) \\
& =\int \tilde{W}\left(\xi_{1}, \eta_{1} ; \xi_{2}, \eta_{2}\right) \exp \left[-\mathrm{i}\left(\xi_{1} q_{1}+\eta_{1} p_{1}+\xi_{2} q_{2}+\eta_{2} p_{2}\right)\right] \frac{d \xi_{1} d \eta_{1} d \xi_{2} d \eta_{2}}{(2 \pi)^{2}} .
\end{aligned}
$$

As one can see all the moments can be obtained by measuring the one-mode optical tomograms as it was shown in examples of quadratic and cubic moments. The procedure to find other highest moments is iterative and starting from the mean values $\left\langle Q_{k}\right\rangle,\left\langle P_{k}\right\rangle,(k=1,2)$, the variances and covariances one obtains all the highest moments by the measured one-mode tomograms of the two-mode $a$ and $b$ light and its symplectically transformed modes $(a \pm b) / \sqrt{2},(a \pm \mathrm{i} b) / \sqrt{2}$. Thus the state of two-mode light is described by one tomogram depending on two random quadratures $X_{1}, X_{2}$ or by a sufficient set of one-mode tomograms $\mathcal{W}_{k}\left(X_{k}, \theta_{k}\right)$ which are appropriate marginals.

\section{Photon statistics}

One can formulate the problem of measuring state in terms of photon statistical properties of the measured two-mode light. For Gaussian states the photon statistics is described by multi-variable Hermite polynomials. For small number of photons, the expressions can be easily constructed. The photon distribution are determined by the highest moments

$$
\left\langle\hat{n}_{1}^{k_{1}} \hat{n}_{2}^{k_{2}}\right\rangle=\operatorname{Tr}\left(\rho \hat{n}_{1}^{k_{1}} \hat{n}_{2}^{k_{2}}\right)
$$


where

$$
\hat{n}_{1}=\frac{1}{2}\left(Q_{1}^{2}+P_{1}^{2}-1\right) ; \hat{n}_{2}=\frac{1}{2}\left(Q_{2}^{2}+P_{2}^{2}-1\right) .
$$

Thus, measuring the photon statistics implies to measure the photon quadrature highest moments. Since highest moments satisfy the quantum uncertainty relations, the photon statistics (quantum correlations) demonstrate difference with properties of classical electromagnetic field. The photon statistics can be studied using measured optical (symplectic) tomograms. Experimental check of the quantum uncertainty relations serves not only to investigate the degree of accuracy with which nowadays the uncertainty relations are known to be fulfilled. Since there are no doubts that the quantum mechanics is a correct theory and the uncertainty relations must be fulfilled, the results of the experiments can serve also to control the correctness of the experimental tools used in homodyne detecting photon states. There exist inequalities in which the highest moments of quadrature components are involved (see, e.g., the review [19]). One can reformulate these highest order inequalities in terms of tomographic quadrature moments given for example in Eq. (67) and to obtain extra inequalities expressed in terms of the experimental values of the optical tomogram. Moreover, we suggested the possibility to use the covariant form of Eq. (67), given by Eq. (69), more suitable for an experimental check. The tomographic probability approach can be applied also for two-mode and multi-mode photon states especially for Gaussian states for which their properties like photon statistics are sufficiently known.

Thus, the photon distribution function for the two-mode field is explicitly given in 22] in terms of Hermite polynomials of four variables, related to quadrature variances and covariances of the Gaussian field states.

Thus, measuring both photon statistics and optical tomograms provides the possibility of a cross checking of the quantum inequalities for the quadrature highest moments.

\section{Conclusions}

To summarize, we list the main results of this paper. For two-mode quantum field we express the photon quadrature uncertainty relations, like the Robertson's ones, in terms of measurable optical tomograms of one-mode quantum electromagnetic field. We suggest to use the given tomographic 
expression of the Robertson's inequality to control the accuracy of the homodyne photon state detection. Also, we give examples of inequalities for highest moments of the photon quadratures for one-mode field. We have expressed all the inequalities in tomographic form, in particular in covariant form, this is suitable for experimental checking. Such checking, to the best of our knowledge, has not yet been done due to the absence of the technique appropriate for the experimental verification of these basic inequalities, which can be violated in the classical domain. We have connected the checking of the photon statistics to the possible suggested experimental checking of the quadrature statistics. The generalization of the tomographic approach

to study Robertson uncertainty relations to the multi-mode field is shown to be straightforward.

\section{References}

[1] Man'ko V I, Marmo G, Simoni A, Ventriglia F 2009 Adv. Sci. Lett. 2 $517-520$.

[2] Man'ko V I, Marmo G, Porzio A, Solimeno S, and Ventriglia F 2011 Phys.Scr. 83045001.

[3] Schrödinger E 1930 Ber. Kgl. Akad. Wiss., Berlin, 296-303.

[4] Robertson H P 1930 Phys. Rev. 35667.

[5] Bertrand J and Bertrand P 1987 Found. Phys. 17397.

[6] Vogel K and Risken H 1989 Phys. Rev. A 402847.

[7] Smithey D T, Beck M, Raymer M G, Faridani A 1993 Phys. Rev. Lett. 701244.

[8] Mlynek J 1996 Phys. Rev. Lett. 772933.

[9] Lvovsky A I, Hansen H, Alchele T, Benson O, Mlynek J, and Schiller S 2001 Phys. Rev. Lett. 87050402.

[10] Parigi V, Zavatta A, Kim M, Bellini A et al. 2007 Science 3171980.

[11] D'Auria V, Fornaro S, Porzio A, Solimeno S, Olivares S, and Paris M G A 2009 Phys. Rev. Lett. 102020502. 
[12] Mancini S, Man'ko V I and Tombesi P 1996 Phys. Lett. A 2131.

[13] Man'ko V I, Marmo G, Simoni A, Stern A, Ventriglia F 2005 Phys. Lett. A 343251.

[14] Man'ko V I, Marmo G, Simoni A, Stern A, Sudarshan E C G, Ventriglia F 2006 Phys. Lett. A 3511.

[15] Man'ko V I, Marmo G, Simoni A, Ventriglia F 2006 Open Sys. and Information Dyn. 13239.

[16] Man'ko O V and Man'ko V I 1997 J. Russ. Laser Res. 18407.

[17] D'Ariano G M, Mancini S, Man'ko V I and Tombesi P 1996 Quantum Semiclass. Opt. 81017

[18] Ibort A, Man'ko V I, Marmo G, Simoni A, Ventriglia F 2009 Phys. Scr. 79 065013-065041.

[19] Dodonov V V, Manko V I 1989 Proc. of Lebedev Physical Inst. Vol. 183 (New York, Nova science).

[20] Dodonov V V, Kurmyshev E V, Manko V I 1980 Phys. Lett. A 79150.

[21] Bhamathi G, Chiu C B, Sudarshan E C G 1995 Phys. Rev. A 52 43-54.

[22] Dodonov V V, Man'ko O V and Man'ko V I 1994 Phys. Rev. A 50813.

[23] Robertson H P 1934 Phys. Rev. 46794.

[24] Ibort A, Man'ko V I, Marmo G, Simoni A, Ventriglia F 2009 J. Phys A: Math. Theor. 42 155302-155313, 